\title{
Efeitos do extrato hidroalcoólico das folhas e da farinha dos frutos de Guabiju (Myrcianthes pungens) sobre parâmetros laboratoriais de ratos diabéticos
}

Effects of the hydroalcoholic extract from the leaves and flour of the fruits of Guabiju (Myrcianthes pungens) on laboratory parameters of diabetic rats

Efectos del extracto hidroalcohólico de las hojas y harina de los frutos de Guabiju (Myrcianthes pungens) sobre parámetros de laboratorio de ratas diabéticas

Recebido: 22/06/2021 | Revisado: 30/06/2021 | Aceito: 01/07/2021 | Publicado: 14/07/2021

Amanda Batista da Silva Lemos ORCID: https://orcid.org/0000-0002-0938-8289 Universidade José do Rosário Vellano, Brasil E-mail: amanda.bsl@outlook.com

Amanda Ribeiro da Fonseca ORCID: https://orcid.org/0000-0001-6509-0404 Universidade José do Rosário Vellano, Brasil E-mail: amanda.rbf27@yahoo.com.br

Ana Elisa Silveira de Souza

ORCID: https://orcid.org/0000-0001-5416-6047 Universidade José do Rosário Vellano, Brasil

E-mail: anaelisa_giuliano@hotmail.com

Luiza Bim Vivan

ORCID: https://orcid.org/0000-0003-4210-8485 Universidade José do Rosário Vellano, Brasil E-mail: luizabvivian@ outlook.com

Laryssa Aparecida Oliveira Lomonaco ORCID: https://orcid.org/0000-0003-3976-0624 Universidade José do Rosário Vellano, Brasil

E-mail: laryssalomonaco@hotmail.com

Sara Tais Dias

ORCID: https://orcid.org/0000-0001-7826-3546 Universidade José do Rosário Vellano, Brasil

E-mail: sarataisdias@outlook.com

Bruno Cesar Correa Salles

ORCID: https://orcid.org/0000-0002-4444-9673 Universidade José do Rosário Vellano, Brasil E-mail: bruno.alfenas@hotmail.com

Cláudio Daniel Cerdeira

ORCID: https://orcid.org/0000-0002-7242-8028 Universidade Federal de Alfenas, Brasil E-mail: daniel.cerdeira.84@gmail.com

Maria Tereza Bernardes Pascoal ORCID: https://orcid.org/0000-0002-3032-030X Universidade José do Rosário Vellano, Brasil E-mail: maria.bernardes@unifenas.br

Rafaela Bergmann Strada de Oliveira ORCID: https://orcid.org/0000-0001-5260-2183 Universidade José do Rosário Vellano, Brasil E-mail: rafaela.bergmann@ unifenas.br

Gérsika Bitencourt Santos Barros ORCID: https://orcid.org/0000-0003-0849-2786 Universidade José do Rosário Vellano, Brasil E-mail: gersikasantos@unifenas.br

\section{Resumo}

O Diabetes Mellitus (DM) é um sério problema de saúde pública que carece de novas alternativas terapêuticas, dentre estas, destaca-se a fitoterapia. Esse estudo teve como objetivo avaliar a ação do extrato hidroetanólico das folhas e da farinha dos frutos de Guabiju (Myrcianthes pungens) sobre os parâmetros laboratoriais de animais diabéticos. A farinha dos frutos de Guabiju incorporada na alimentação diária em forma de ração peletizada ou o extrato das folhas de Guabiju administrado por gavagem (dose oral de $300 \mathrm{mg} / \mathrm{kg} / \mathrm{dia}$ ), foram administrados em um modelo 
experimental de ratos Wistar com DM tipo 1 induzido por aloxano, durante 45 e 90 dias, respectivamente. Avaliou-se o efeito anti-hiperglicemiante e outros parâmetros bioquímicos, como triglicérides, colesterol total e enzimas marcadoras de função hepática (AST) e renal (creatinina). O desfecho de ambos os tratamentos não culminou em marcante diminuição da glicemia de jejum, melhora do perfil lipídico ou função renal quando comparado aos ratos diabéticos não tratados. No entanto, o tratamento com o extrato das folhas de Guabiju preveniu o aumento de marcadores de lesão hepática, sendo que os resultados da prova de função hepática mostraram alterações benéficas significativas nos animais tratados com o extrato das folhas de Guabiju, com redução dos níveis de AST. O valor de AST para os ratos não diabéticos foi 72,12 $\pm 10,52 \mathrm{mg} / \mathrm{dl}$, para os ratos diabéticos foi 111,0 $\pm 16,84 \mathrm{mg} / \mathrm{dl} \mathrm{e} \mathrm{para} \mathrm{os}$ ratos diabéticos tratados foi 82,66 $\pm 5,50 \mathrm{mg} / \mathrm{dl}(p<0,05)$. Esta ação em prevenir típicas complicações do DM, como os danos hepáticos, em uma baixa dose, demonstra que esta planta pode apresentar uma possível atividade terapêutica, que deve ser refinada com mais testes e ensaios clínicos que comprovem uma eficácia da ação e segurança.

Palavras-chave: Diabetes mellitus; Myrcianthes pungens; Plantas medicinais; Creatinina; Perfil lipídico; AST.

\begin{abstract}
Diabetes Mellitus (DM) is a serious public health problem that lacks new therapeutic alternatives, among which, phytotherapy stands out. This study aimed to evaluate the activity of hydroethanolic extract of leaves and flour from Guabiju fruits (Myrcianthes pungens) on the laboratory parameters of diabetic animals. The flour of the Guabiju fruits incorporated into the daily diet in the form of pelleted feed or the extract of the Guabiju leaves administered by gavage (oral dose of $300 \mathrm{mg} / \mathrm{kg} / \mathrm{day}$ ), were administered in an experimental model of Wistar rats with type 1 DM induced by alloxane, for 45 and 90 days, respectively. The anti-hyperglycemic effect and other biochemical parameters were evaluated, such as triglycerides, total cholesterol, and liver (AST) and renal (creatinine) function marker enzymes. The outcome of both treatments did not result in a marked decrease in fasting glycemia, improvement of the lipid profile or renal function when compared to untreated diabetic rats. However, treatment with Guabiju leaf extract prevented the increase in liver injury, and the results of the hepatic function showed significant beneficial changes in animals treated with Guabiju leaf extract, with decreased levels of AST. The AST value for nondiabetic rats was $72.12 \pm 10.52 \mathrm{mg} / \mathrm{dl}$, for diabetic rats it was $111.0 \pm 16.84 \mathrm{mg} / \mathrm{dl}$ and for treated diabetic rats it was $82.66 \pm 5.50 \mathrm{mg} / \mathrm{dl}(\mathrm{p}<0.05)$. This action in preventing typical complications of $\mathrm{DM}$, such as liver damage, in a low dose, demonstrates that this plant can present a possible therapeutic activity, which must be refined with further tests and clinical trials that prove its effectiveness and safety.
\end{abstract}

Keywords: Diabetes mellitus; Myrcianthes pungens; Medicinal plants; Creatinine; Lipid profile; AST.

\title{
Resumen
}

La Diabetes Mellitus (DM) es un grave problema de salud pública que carece de nuevas alternativas terapéuticas, entre las que destaca la fitoterapia. Este estudio tuvo como objetivo evaluar la acción del extracto hidroetanólico de las hojas y harina de los frutos de Guabiju (Myrcianthes pungens) sobre los parámetros de laboratorio de animales diabéticos. Se administró harina de fruta de guabiju incorporada a la dieta diaria en forma de pienso granulado o el extracto de hojas de guabiju administrado por sonda (dosis oral de $300 \mathrm{mg} / \mathrm{kg} /$ día) en un modelo experimental de ratas Wistar con DM tipo 1 inducida por aloxano, durante 45 y 90 días, respectivamente. Se evaluó el efecto antihiperglucémico y otros parámetros bioquímicos, como triglicéridos, colesterol total y enzimas marcadores de función hepática (AST) y renal (creatinina). El resultado de ambos tratamientos no culminó en una disminución marcada de la glucosa en ayunas, una mejora en el perfil de lípidos o la función renal en comparación con las ratas diabéticas no tratadas. Sin embargo, el tratamiento con extracto de hoja de Guabiju evitó el aumento de los marcadores de daño hepático, y los resultados de la prueba de función hepática mostraron cambios beneficiosos significativos en los animales tratados con extracto de hoja de Guabiju, con niveles reducidos de AST. El valor de AST para ratas no diabéticas fue $72,12 \pm 10,52 \mathrm{mg} / \mathrm{dl}$, para ratas diabéticas fue 111,0 $\pm 16,84 \mathrm{mg} / \mathrm{dl} \mathrm{y}$ para ratas diabéticas tratadas fue $82,66 \pm 5,50 \mathrm{mg} / \mathrm{dl}(p<0,05)$. Esta acción en la prevención de complicaciones típicas de la DM, como el daño hepático, en dosis bajas, demuestra que esta planta puede presentar una posible actividad terapéutica, la cual debe refinarse con más pruebas y ensayos clínicos que demuestren una acción eficaz y segura. Palabras clave: Diabetes mellitus; Myrcianthes pungens; Plantas medicinales; Creatinina; Perfil lipídico; AST.

\section{Introdução}

O Diabetes Mellitus é um grupo vasto e diversificado de distúrbios metabólicos, que tem como característica comum à hiperglicemia prolongada, resultante de defeitos na ação da insulina, na secreção de insulina, ou nos receptores insulínicos (Silva et al., 2016; Brito et al., 2019). Segundo as novas diretrizes da Sociedade Brasileira de Diabetes (2017-2018), em humanos, o diagnóstico de DM é estabelecido através de glicemia de jejum persistente $\geq 126 \mathrm{mg} / \mathrm{dl}$ ou glicemia pós prandial 
persistente após sobrecarga com 75 gramas de glicose $\geq 200 \mathrm{mg} / \mathrm{dl}$ ou glicemia ao acaso persistente $\geq 200 \mathrm{mg} / \mathrm{dl}$ associado com sintomas como poliúria, polidipsia, polifagia e emagrecimento, além da hemoglobina glicada $\geq 6.5 \%$ (SBD, 2019/2020).

A hiperglicemia crônica no DM não controlado é responsável por complicações microvasculares e macrovasculares no organismo, gerando retinopatia, nefropatia, neuropatia, doença coronariana, doença cerebrovascular e doença arterial periférica (Silva et al., 2016; Brito et al., 2019; Silva et al., 2019). Uma vez que a glicemia alterada é considerada uma variável contínua de risco, o tratamento comportamental e medicamentoso visando se estabelecer uma normoglicemia consiste em principais meios de reduzir as complicações à longo prazo no DM (SBD, 2018).

Carente de novas, eficazes e seguras terapias medicamentosas, o DM apresenta alta incidência mundial, afetando todas as classes sociais. Nesse contexto, o uso de plantas medicinais para o tratamento e prevenção de diversas doenças é uma prática antiga, sendo utilizada desde os primórdios da civilização, além de que diversos estudos te investigado as mais variadas bioatividades de plantas (Filho et al., 2021). No Brasil, esse hábito é favorecido visto a enorme riqueza e variedade de espécies da flora nativa, podendo complementar tratamentos, principalmente, voltado às populações de baixa renda (Coan \& Matias, 2013). De acordo com Souza e Romagnolo (2004), a espécie Myrcianthes pungens, popularmente conhecida como Guabiju, guabiroba-açú, guabijueiro, guabira-guaçu, ibariu ou ibaviu, é nativa da Bacia do Prata. Seus frutos são pequenos e de coloração púrpura ou roxo-avermelhada, com utilidade na medicina popular devido as suas propriedades antidisentéricas e antioxidantes, estas últimas, graças à presença de carotenoides e antocianinas, demonstrando potencial para uso farmacológico (Apel et al., 2006; Nora, 2012). Suas folhas são pequenas, simples, com presença de pecíolo, de bordos completos e, quando maduras, possuem cor verde escura e acúleo na ponta. É relatado o uso das folhas como xarope e elas apresentam atividade disentérica, além de regular a função intestinal e serem taníferas (Rodrigues et al., 2016).

Diante do até aqui exposto, esse estudo teve como objetivo avaliar a ação do extrato hidroetanólico das folhas de Guabiju e da farinha dos frutos, sobre os parâmetros laboratoriais (glicemia, perfil lipídico e funções hepática e renal) em um modelo experimental de animais com DM tipo 1 induzido por Aloxano.

\section{Metodologia}

\section{Aspectos éticos}

Todos os experimentos envolvendo animais foram conduzidos de acordo com as recomendações do "Guide for the Care and Use of Laboratory Animals" (TNAP, 2011). Este estudo foi previamente aprovado pelo comitê de ética institucional sobre o uso de animais (parecer $n^{\circ} 53 \mathrm{~A} / 2015$ ). O estudo não envolveu qualquer espécie ameaçada de extinção e/ou protegida e nenhuma permissão específica foi requerida quanto ao uso do material vegetal.

\section{Obtenção da farinha dos frutos de guabiju}

A farinha do fruto de Guabiju foi obtida da empresa Quinta das Laranjeiras Alimentos Inteligentes LTDA especializada em desenvolvimento e comercialização de produtos e matérias-primas naturais no estado do Rio Grande do Sul (RS). A farinha foi adicionada à ração comercial para obtenção de uma mistura com uma concentração da farinha igual à $10 \%$.

\section{Obtenção do extrato hidroetanólico das folhas de guabiju}

As folhas de Myrcianthes pungens foram obtidas da empresa Quinta das Laranjeiras Alimentos Inteligentes LTDA, arranjadas em camadas e submetidas à secagem em estufa com circulação de ar a $45^{\circ} \mathrm{C}$ até a completa secagem (quando o peso constante de $10 \mathrm{~g}$ foi alcançado) e pulverizadas em moinho de facas. $\mathrm{O}$ extrato hidroetanólico foi obtido através do método de maceração, de acordo com Silva et al. (2019). Após o intumescimento de $500 \mathrm{~g}$ do pó das folhas com o líquido extrator (álcool: 
água $7: 3 \mathrm{v} / \mathrm{v}$ ) durante 30 minutos, fora do percolado, mais extrator (álcool $70 \%$ ) foi empacotado com a mistura. $\mathrm{O}$ empacotamento foi feito de uma forma mais homogênea possível, evitando a formação de bolhas ou buracos no conteúdo alocado dentro. O álcool 70\% foi gradualmente adicionado até cobrir o pó e a mistura foi deixada por 21 dias em maceração, com agitação diária. Após esta etapa, a mistura foi submetida ao processo de rota-evaporação sob uma pressão reduzida (500 $\mathrm{mmHg}$ ) e temperatura de $50^{\circ} \mathrm{C}$ para concentrar o extrato (e eliminar o álcool) e, finalmente, liofilizada para completa remoção da água. O extrato seco obtido foi apropriadamente armazenado e, quando do uso, solubilizado em água destilada para administração por gavagem aos animais.

\section{Animais e grupos experimentais}

$\mathrm{O}$ trabalho foi realizado em dois momentos (sets de experimentos) (Tabela 1). No primeiro momento foram utilizados 24 ratos machos de 6 a 9 semanas da linhagem Wistar com peso corporal inicial próximo à $300 \mathrm{~g}$. Os ratos foram divididos em 3 grupos experimentais, sendo eles: não diabéticos controle (se alimentaram com ração comercial normal), diabéticos controle (se alimentaram com ração comercial normal) e diabéticos tratados (se alimentaram com ração comercial contendo $10 \%$ de farinha de Guabiju). O tratamento foi realizado por 45 dias.

No segundo momento foram utilizados 20 ratos machos de 6 a 9 semanas da linhagem Wistar com peso corporal inicial próximo de $300 \mathrm{~g}$. Os ratos foram divididos em 3 grupos experimentais, sendo eles: controle não diabéticos, controle diabéticos e diabéticos tratados com extrato hidroetanólico das folhas de Guabiju em uma concentração de $300 \mathrm{mg} / \mathrm{Kg}$. O tratamento foi realizado por 90 dias.

Tabela 1 - Representação dos grupos experimentais.

\section{$1^{\circ}$ SET DE EXPERIMENTOS (Farinha dos frutos)}

\begin{tabular}{|c|c|c|c|}
\hline Grupo experimental & $\mathbf{N}^{\mathbf{o}}$ de animais & Indução do DM & $\begin{array}{l}\text { Tratamento } \\
\text { (45 dias) }\end{array}$ \\
\hline Controle não diabéticos & 8 & - & Solução salina \\
\hline Controle diabéticos & 8 & Aloxana $130 \mathrm{mg} / \mathrm{Kg}$ & Solução salina \\
\hline Diabéticos tratados com Guabiju & 8 & Aloxana $130 \mathrm{mg} / \mathrm{Kg}$ & $\begin{array}{l}\text { Farinha dos frutos de } \\
\text { Guabiju } 10 \%\end{array}$ \\
\hline \multicolumn{4}{|c|}{$2^{\circ}$ SET DE EXPERIMENTOS (Extrato hidroetanólico das folhas) } \\
\hline Grupo experimental & $N^{o}$ de animais & Indução do DM & $\begin{array}{l}\text { Tratamento } \\
\text { (90 dias) }\end{array}$ \\
\hline Controle não diabéticos & 8 & - & Solução salina \\
\hline Controle diabéticos & 6 & Aloxana $130 \mathrm{mg} / \mathrm{Kg}$ & Solução salina \\
\hline Diabéticos tratados com Guabiju & 6 & Aloxana $130 \mathrm{mg} / \mathrm{Kg}$ & $\begin{array}{l}\text { Extrato } \\
\text { hidroetanólico das } \\
\text { folhas de Guabiju } \\
(300 \mathrm{mg} / \mathrm{Kg} / \mathrm{dia})\end{array}$ \\
\hline
\end{tabular}

Fonte: Autores. 


\section{Indução do diabetes experimental}

Em ambos os sets de experimentos foi utilizado a droga diabetogênica Aloxano na dose de $130 \mathrm{mg} / \mathrm{Kg}$ de peso corporal, dissolvido em salina $0,9 \%(\mathrm{pH} 4,5)$, por via intraperitoneal para induzir o DM tipo 1, conforme metodologia previamente preconizadas (Brito et al., 2019; Silva et al., 2019). Os animais que apresentaram glicemia, medida por punção capilar da cauda, acima de $200 \mathrm{mg} / \mathrm{dL}$, foram considerados diabéticos. Os níveis de glicose e o peso dos animais foram avaliados uma semana após a indução do DM, para saber se os animais estavam diabéticos e iniciar a dieta (a glicose sanguínea foi avaliada com um glicosímetro comercial usando sangue da veia da cauda).

\section{Coleta do material e eutanásia}

Após o período de tratamento, 45 ou 90 dias, os animais foram mantidos em jejum por 12 horas e em seguida anestesiados usando Isoflurano a 5\% e o sangue foi coletado por punção cardíaca. O método de indução de morte foi por aprofundamento anestésico para depressão bulbar, e, após isso, os animais foram armazenados em sacos plásticos com capacidade e resistência compatíveis com o peso, que foram identificados e mantidos em freezer até a coleta por pessoal treinado que levou ao desfecho final (incineração).

\section{Avaliação dos Parâmetros bioquímicos}

Os ratos de todos os grupos foram deixados em jejum por um período de 12 horas. Após esse período, o sangue coletado foi centrifugado a $1500 \mathrm{rpm}$ durante 10 minutos. O soro obtido foi utilizado para quantificação da glicose de jejum, triglicérides, colesterol total e enzimas marcadoras de função hepática (AST) e renal (creatinina), através de análises laboratoriais padronizadas, como relatado em prévios estudos (Silva et al., 2016; Brito et al., 2019; Silva et al., 2019).

\section{Análise estatística}

Todos os resultados foram expressos como média mais ou menos o desvio padrão de, no mínimo, três experimentos realizados em triplicata. Os resultados foram submetidos ao teste da ANOVA e o teste de Tukey para comparações múltiplas das médias. Valores de $p$ iguais ou menores que $0,05(\alpha=5 \%)$ entre as diferenças encontradas, foram considerados estatisticamente significativos.

\section{Resultados}

Em relação à glicemia de jejum dos ratos tratados com farinha dos frutos de Guabiju (Tabela 2), os animais não diabéticos apresentaram glicemia de 90,6 $\pm 8,23 \mathrm{mg} / \mathrm{dl}$, os diabéticos tiveram glicemia de $205 \pm 15,03 \mathrm{mg} / \mathrm{dl}$ e os ratos diabéticos tratados tiveram glicemia de $218 \pm 42,01 \mathrm{mg} / \mathrm{dl}$. A farinha de Guabiju a $10 \%$ não interferiu significativamente nos valores de glicemia dos ratos do grupo diabético tratado em relação ao grupo diabético controle. Quando os animais foram tratados com o extrato hidroetanólico das folhas de Guabiju (300 mg/Kg), os resultados mostraram que os ratos não diabéticos apresentaram glicemia de 108,1 $\pm 25,62 \mathrm{mg} / \mathrm{dl}$, os diabéticos tiveram glicemia de 459,2 $\pm 91,95 \mathrm{mg} / \mathrm{dl}$ e os ratos diabéticos tratados tiveram glicemia de 490,0 $\pm 32,14 \mathrm{mg} / \mathrm{dl}$. O extrato das folhas de Guabiju também não interferiu significativamente nos valores de glicemia dos ratos do grupo diabético tratado em relação ao grupo diabético controle.

Em relação ao perfil lipídico (Tabela 3) dos animais tratados com farinha dos frutos de Guabiju, os ratos não diabéticos apresentaram triglicérides de $89,1 \pm 10,9 \mathrm{mg} / \mathrm{dl}$ e colesterol total de $53,4 \pm 4,25 \mathrm{mg} / \mathrm{dl}$; os diabéticos tiveram triglicérides de 65,3 $\pm 4,06 \mathrm{mg} / \mathrm{dl}$ e colesterol total de $53,8 \pm 3,95 \mathrm{mg} / \mathrm{dl} \mathrm{e}$ os ratos diabéticos tratados tiveram triglicérides de $68,8 \pm 5,56 \mathrm{mg} / \mathrm{dl}$ e colesterol total de $56,2 \pm 5,42 \mathrm{mg} / \mathrm{dl}$. Demonstrando assim que a farinha dos frutos de Guabiju não 
beneficiou o perfil lipídico dos ratos (triglicerídeos, colesterol total e HDL), porém os ratos tratados com a farinha de Guabiju não pioraram o seu perfil lipídico, sendo que todos os resultados dos ratos diabéticos foram estatisticamente iguais aos dos ratos tratados. Quando os ratos foram tratados com o extrato hidroetanólico das folhas da planta, os animais não diabéticos apresentaram triglicérides de $25,5 \pm 16,1 \mathrm{mg} / \mathrm{dl}$ e colesterol total de $60,5 \pm 18,7 \mathrm{mg} / \mathrm{dl}$; os diabéticos tiveram triglicérides de $70,4 \pm 26,7 \mathrm{mg} / \mathrm{dl}$ e colesterol total de $67,4 \pm 17,1 \mathrm{mg} / \mathrm{dl}$ e os ratos diabéticos tratados tiveram triglicérides de 87,0 $\pm 28,3$ $\mathrm{mg} / \mathrm{dl}$ e colesterol total de 73,3 $\pm 15,5 \mathrm{mg} / \mathrm{dl}$. Demonstrando novamente que o extrato das folhas de Guabiju não beneficiou o perfil lipídico dos ratos (triglicerídeos e colesterol total).

Com relação às provas de função renal (Tabela 4) dos animais tratados com a farinha do fruto, a creatinina dos ratos não diabéticos apresentou o valor de $0,56 \pm 0,03 \mathrm{mg} / \mathrm{dl}$; os diabéticos $0,75 \pm 0,05 \mathrm{mg} / \mathrm{dl}$ e os ratos diabéticos tratados $0,69 \pm$ $0,04 \mathrm{mg} / \mathrm{dl}$. Nos animais tratados com o extrato das folhas de Guabiju o resultado foi semelhante, pois, a creatinina dos ratos não diabéticos apresentou o valor de $0,52 \pm 0,06 \mathrm{mg} / \mathrm{dl}$; os diabéticos $0,88 \pm 0,11 \mathrm{mg} / \mathrm{dl}$ e os ratos diabéticos tratados $0,89 \pm$ $0,11 \mathrm{mg} / \mathrm{dl}$.

Nas provas de função hepática (Tabela 5) do grupo tratado com a farinha dos frutos, o valor de Aspartato Transaminase (AST) para os ratos não diabéticos foi $31 \pm 4,20 \mathrm{mg} / \mathrm{dl}$, para os ratos diabéticos foi $44 \pm 4,10 \mathrm{mg} / \mathrm{dl}$ e para os ratos diabéticos ratados foi $48 \pm 3,44 \mathrm{mg} / \mathrm{dl}$. Entretanto, os resultados da prova de função hepática mostraram alterações benéficas significativas nos animais tratados com o extrato das folhas de Guabiju, com redução dos níveis de AST. O valor de AST para os ratos não diabéticos foi $72,12 \pm 10,52 \mathrm{mg} / \mathrm{dl}$, para os ratos diabéticos foi $111,0 \pm 16,84 \mathrm{mg} / \mathrm{dl}$ e para os ratos diabéticos tratados foi $82,66 \pm 5,50 \mathrm{mg} / \mathrm{dl}$.

Tabela 2 - Glicemia de jejum dos animais.

\begin{tabular}{llcc}
\hline \multicolumn{1}{c}{ Grupos } & \multicolumn{3}{c}{ Glicemia (mg/dl) } \\
\cline { 2 - 3 } & Sem DM & Diabético & Tratado \\
\hline $1^{\circ}$ set de experimentos: & $90,6 \pm 8,23^{\mathrm{a}}$ & $205 \pm 15,03^{\mathrm{b}}$ & $218 \pm 42,01^{\mathrm{b}}$ \\
\hline $\begin{array}{l}\text { Farinha dos frutos de Guabiju 10\% } \\
2^{\mathbf{o}} \text { set de experimentos: }\end{array}$ & $108,1 \pm 25,62^{\mathrm{a}}$ & $459,2 \pm 91,95^{\mathrm{b}}$ & $490,0 \pm 32,14^{\mathrm{b}}$ \\
\hline
\end{tabular}

Fonte: Autores.

Tabela 3 - Resultados do perfil lipídico dos animais.

\begin{tabular}{lllllll}
\hline & \multicolumn{3}{c}{ Triglicerídeos (mg/dl) } & \multicolumn{2}{c}{ Colesterol Total (mg/dl) } \\
\cline { 2 - 7 } Grupos & Sem DM & Diabético & Tratado & Sem DM & Diabético & Tratado \\
\hline $\begin{array}{l}\text { Farinha dos frutos } \\
\text { de Guabiju 10\% }\end{array}$ & $89,1 \pm 10,9^{\mathrm{a}}$ & $65,3 \pm 4,06^{\mathrm{a}}$ & $68,8 \pm 5,56^{\mathrm{a}}$ & $53,4 \pm 4,25^{\mathrm{a}}$ & $53,8 \pm 3,95^{\mathrm{a}}$ & $56,2 \pm 5,42^{\mathrm{a}}$ \\
\hline $\begin{array}{l}\text { Extrato das folhas de } \\
\text { Guabiju 300 mg/Kg }\end{array}$ & $25,5 \pm 16,1^{\mathrm{a}}$ & $70,4 \pm 26,7^{\mathrm{b}}$ & $87,0 \pm 28,3^{\mathrm{b}}$ & $60,5 \pm 18,7^{\mathrm{a}}$ & $67,4 \pm 17,1^{\mathrm{a}}$ & $73,3 \pm 15,5^{\mathrm{a}}$ \\
\hline
\end{tabular}

Fonte: Autores. 
Tabela 4 - Prova de função renal (creatinina).

\begin{tabular}{llcc}
\hline \multirow{2}{*}{ Grupos } & \multicolumn{3}{c}{ Creatinina (mg/dl) } \\
\cline { 2 - 4 } & \multicolumn{1}{c}{ Sem DM } & Diabético & Tratado \\
\hline $\begin{array}{l}1^{\circ} \text { set de experimentos: } \\
\text { Farinha dos frutos de Guabiju 10\% }\end{array}$ & $0,56 \pm 0,03^{\mathrm{a}}$ & $0,75 \pm 0,05^{\mathrm{b}}$ & $0,69 \pm 0,04^{\mathrm{b}}$ \\
\hline $\begin{array}{l}2^{\mathrm{o}} \text { set de experimentos: } \\
\text { Extrato das folhas de Guabiju 300 } \mathrm{mg} / \mathrm{Kg}\end{array}$ & $0,52 \pm 0,06^{\mathrm{a}}$ & $0,88 \pm 0,11^{\mathrm{b}}$ & $0,89 \pm 0,11^{\mathrm{b}}$ \\
\hline
\end{tabular}

Fonte: Autores.

Tabela 5 - Resultado da prova de função hepática (AST)

\begin{tabular}{llll}
\hline \multicolumn{1}{c}{ Grupos } & \multicolumn{3}{c}{ AST (mg/dl) } \\
\cline { 2 - 4 } & \multicolumn{1}{c}{ Sem DM } & Diabético & Tratado \\
\hline $\begin{array}{l}1^{\circ} \text { set de experimentos: } \\
\text { Farinha dos frutos de Guabiju 10\% }\end{array}$ & $31 \pm 4,20^{\mathrm{a}}$ & $44 \pm 4,10^{\mathrm{b}}$ & \multirow{2}{*}{$48 \pm 3,44^{\mathrm{b}}$} \\
\hline $\begin{array}{l}2^{\circ} \text { set de experimentos: } \\
\text { Extrato das folhas de Guabiju 300 } \mathrm{mg} / \mathrm{Kg}\end{array}$ & $72,12 \pm 10,52^{\mathrm{a}}$ & $111,0 \pm 16,84^{\mathrm{b}}$ & $82,66 \pm 5,50^{\mathrm{a}}$ \\
\hline
\end{tabular}

Fonte: Autores.

\section{Discussão}

O atual arsenal terapêutico disponível para o tratamento do DM permite uma melhora nos sintomas, a melhoria da qualidade de vida e uma diminuição do risco de complicações crônicas através de um controle glicêmico adequado (Hissa et al., 2001). No presente estudo mostramos que tanto a farinha dos frutos quanto o extrato das folhas de Guabiju não tiveram capacidade de diminuir significativamente os valores de glicemia em ratos diabéticos. Nora et al. (2014) mostraram que o Guabiju apresenta pouca efetividade em diminuir a glicemia de animais tratados, atribuindo que a alta concentração de açúcar presente nesta planta não contribui na diminuição dos níveis de glicose sanguínea.

Sendo a síndrome metabólica uma complicação frequente do DM não controlado, geneticamente condicionado e relacionado aos hábitos de vida dos pacientes, seria um importante passo no tratamento da doença, a melhoria do perfil lipídico (Gonçalves et al., 2017). Isso é conquistado com medicações habituais, dieta e exercício físico (SBD, 2018). No presente estudo a farinha dos frutos e o extrato das folhas de Guabiju não conduziram a melhorias no perfil lipídico dos ratos, uma vez que primariamente estes tratamentos não preveniram a hiperglicemia, e desde que, em certos casos (excetuando-se causas que cursam com dislipidemia primária ou paralela ao DM, como exemplo, a síndrome metabólica), a dislipidemia pode ser uma complicação do DM não controlado (Silva et al., 2016; Brito et al., 2019; Silva et al., 2019). Tal achado difere do encontrado por Nora et al. (2014) e por Leontowicz et al. (2007), em que os ratos com estresse oxidativo induzido por cisplatina tiveram uma redução das taxas de CT, LDL e TG, apesar de não apresentarem aumento do HDL. Como o Guabiju é um fruto comprovadamente hiperproteico, a diminuição do perfil lipídico no último estudo pode estar relacionada à presença de aminoácidos que inibem especificamente a citotoxidade da cisplatina, uma vez que a quantidade menor de lipídeos composta por fontes antioxidantes não justificou essa redução no primeiro estudo (Leontowicz et al., 2007; Nora et al., 2014).

Como uma consideração a ser feita, no presente estudo, os ratos diabéticos não apresentaram valores de colesterol e triglicérides compatíveis com uma provável dislipidemia de acordo com o grupo controle. O fato é que a manutenção dos valores do perfil lipídico consiste em um grande avanço visto que pode ser considerado um fator estabilizador das complicações trazidas pelo diabetes.

A hiperglicemia crônica pode causar lesões glomerulares. Como prevenção, a redução nos valores de glicemia mantendo-os próximo do limite de normalidade evita complicações crônicas como as lesões renais, reafirmando a importância 
de um tratamento adequado. $\mathrm{O}$ fator desencadeante da lesão renal poderia ser o aumento do estresse oxidativo encontrado no DM (SBD, 2018). De fato, diversos produtos antioxidantes vêm sendo estudados na tentativa de impedir o estresse oxidativo associado as complicações do DM, dentre estes o Guabiju, grande parte deles atuando diretamente em conter danos oxidativos e avaliados por diminuição de lesões diretas (Silva et al., 2016; Brito et al., 2019; Silva et al., 2019). A caracterização química do Guabiju revelou que esta planta apresenta antioxidantes (Detoni, 2015). Nesse contexto, uma vez que os tratamentos aqui performados não apresentaram efeito anti-hiperglicemiante, a prevenção de complicações crônicas do DM não controlado foi ineficaz, tais como a prevenção no aumento dos níveis de creatinina, desde que não se observou essa melhora no valor da prova de função renal dos ratos tratados com a farinha dos frutos ou extrato das folhas de Guabiju. No estudo conduzido por Nora et al. (2014) não houve danos aos rins, induzido pela cisplatina, não havendo alterações nos valores de creatinina que pudessem ser comparados com o presente estudo. Em contrapartida, corroborando com o presente estudo, um estudo dirigido por Yousef et al. (2009) com o extrato de semente de uva apresentou redução do nível de creatinina indicando proteção, mas quando comparado com o grupo controle não se observou diferenças significativas.

Por fim, no DM, principalmente o tipo 2, é relatado uma alta incidência de esteatose hepática por conta de um acúmulo de lipídios nos hepatócitos ocasionados por uma hiperinsulinemia compensatória com inadequada metabolização de ácidos graxos (Cruz et al., 2016). Em concordância com estudo realizado por Yousef et al. (2009), com extrato de semente de uva sobre os efeitos da cisplatina em ratos, houve prevenção no aumento dos níveis plasmáticos de AST quando foi administrado aos ratos o extrato das folhas de Guabiju. Diferente do que aconteceu com a administração da farinha dos frutos de Guabiju. Este resultado pode ser justificado através da maior concentração de fibras na região das folhas do que nos frutos, as fibras nesse caso, atuariam na redução da deposição de gordura no fígado e não especificamente na redução da glicemia ou sobre o estresse oxidativo (Yousef et al., 2009). Ainda, apesar de que na maioria dos casos os antioxidantes estão presentes em maior quantidade nas folhas das plantas comparado aos frutos, esse fato também não influenciou na melhoria de alguns parâmetros bioquímicos aqui avaliados, quando os ratos foram tratados com o extrato das folhas de M. pungens (Guabiju).

\section{Conclusão}

É possível concluir que, apesar da farinha dos frutos e do extrato das folhas do Guabiju não terem melhorado significativamente a glicemia, o perfil lipídico e a função renal (creatinina), houve uma estabilização desses parâmetros, não trazendo malefícios aos animais, frente ao caráter progressivo do DM. Com relação ao extrato das folhas de Guabiju, este preveniu a elevação dos níveis de AST, a uma dose de $300 \mathrm{mg} / \mathrm{kg} / \mathrm{dia}$, evidenciando um possível potencial terapêutico da planta que deve ser mais detalhadamente investigado.

\section{Agradecimentos}

Os autores agradecem à FAPEMIG pelo apoio financeiro.

\section{Referências}

Apel, M. A., et al. (2006). Composição química do óleo volátil de Myrcianthes nativas da região sul do Brasil. Revista Brasileira de Farmacognosia, 16(3), $402-407$.

Brito, A. R., Ravazi, G. F., Oliveira, A. P. P., Cerdeira, C. D., et al. (2019). Nitroxides Improve Biochemical Profiling and Prevent Injuries in the Organs of Type 1 Diabetic Rats. Rev Port Endocrinol Diabetes Metab, 14(2), 128-136. https://doi.org/10.26497/ao180019

Coan, C. M., \& Matias, T. (2013). A utilização das plantas medicinais pela comunidade indígena de Ventarra Alta- RS. Revista de Educação do IDEAU, $8(18), 1-14$

Cruz, J. F., et al. (2016). Relação entre a esteatose hepática não alcoólica e as alterações dos componentes da síndrome metabólica e resistência à insulina. Revista da Sociedade Brasileira de Clínica Médica, 14(2), 79-83. 
Research, Society and Development, v. 10, n. 8, e39910817442, 2021

(CC BY 4.0) | ISSN 2525-3409 | DOI: http://dx.doi.org/10.33448/rsd-v10i8.17442

Detoni, E. Caracterização físico química do Guabiju (Myrcianthes pungues) e métodos de conservação pós colheita. Francisco Beltrão, 2015. 29 f. Trabalho de Conclusão de Curso [Especialização] - Universidade Tecnológica Federal do Paraná.

Filho A. E. F. M. et al. (2021). Hydroetanolic extract activity of Raphanus sativus leaves in submandibular glands of rats with Diabetes Mellitus. Research, Society and Development, 10, 2, p. e21610212447.

Gonçalves, I. C. V., Cerdeira, C. D., Camara, E. P., et al. (2017). Tempol improves lipid profile and prevents left ventricular hypertrophy in LDL receptor gene knockout (LDLr-/-) mice on a high-fat diet. Rev Port Cardiol, 36(9), 629-638. http://dx.doi.org/10.1016/j.repc.2017.02.014

Hissa, M. N., et al. (2001). Tratamento do Diabetes Mellitus tipo 1 com bomba de infusão subcutânea contínua de insulina e insulina lispro. Arquivo Brasileiro de Endocrinologia e Metabologia, 45(5), 487-493.

Leontowicz, M., et al. (2007). Dois frutos exóticos afetam positivamente a composição plasmática do rato. Food Chem, 102(1), 192-200.

Nora, C. D. Caracterização, atividade antioxidante "in vivo" e efeito do processamento na estabilidade de compostos bioativos de araçá vermelho e Guabiju. Porto Alegre, 2012. 91 f. Dissertação [Mestrado em Ciência e Tecnologia de Alimentos] - Universidade Federal do Rio Grande do Sul.

Nora, C. D., et al. (2014). Protective effect of guabiju (Myrcianthes pungens (O. Berg) D. Legrand) and red guava (Psidium cattleyanum sabine) against cisplatin-induced hypercholesterolemia in rats. Brazilian Journal of Pharmaceutical Sciences, 50(3), 483-491.

Rodrigues, M. A., et al. Myrcianthes pungens: caracterização morfológica de folhas e frutos. Relatório técnico-científico apresentado na XXI Jornada de Pesquisa do Salão do Conhecimento Unijui 2016. <https://www.publicacoeseventos.unijui.edu.br/index.php/salaoconhecimento/.../5414>

Romagnolo, M. B. \& souza, M. C. (2004). Os gêneros calycorectes O. Berg, hexachlamys O. berg, myrcianthes o. berg, myrciaria o. Berg e Plinia 1. (Myrtaceae) na planície alagável do alto rio Paraná, Brasil. Acta Botanica Brasilica, 18(3), 613-627.

Silva, A. B., Lopes, G. D. S., Neves, T. V. B., Barros, G. B. S., Reis, L. F. C., Salles, B. C. C., Cerdeira, C. D., Moraes, G. O. I. (2019). Extrato etanólico das folhas de Raphanus sativus L. var. oleífera Metzg (nabo forrageiro): efeitos antihiperglicêmico, antidislipidêmico e antioxidante em ratos com Diabetes Mellitus tipo 1. Revista Fitos. Rio de Janeiro, 13(1), 38-48. http://dx.doi.org/10.17648/2446-4775.2019.654

Silva, A. R., Cerdeira, C. D., Brito, A. R., et al. (2016). Green banana pasta diet prevents oxidative damage in liver and kidney and improves biochemical parameters in type 1 diabetic rats. Arch Endocrinol Metab, 60(4), 355-366. http://dx.doi.org/10.1590/2359-3997000000152

Sociedade Brasileira de Diabetes (SBD). Diretrizes da Sociedade Brasileira de Diabetes $2017-2018 . \quad 383 \quad$ p. <https://www.diabetes.org.br/profissionais/images/2017/diretrizes/diretrizes-sbd-2017-2018.pdf>

The Nacional Academies Press (TNAP). Guide for the care and use of laboratory animals. Ed Eighth. Washinton, DC: 2011. <https://grants.nih.gov/grants/olaw/guide-for-the-care-and-use-of-laboratory-animals.pdf>

Yousef, M. I., Saad, A. A., \& El-Shennawy, L. K. (2009). Efeito protetor do extrato de proantocianidina de semente de uva contra o estresse oxidativo induzido pela cisplatina em ratos. Food Chem. Toxicol, 47(6), 1176-1183. 